\title{
The Development of China's Next Generation Network and National Service Testbed
}

\author{
XU Yanwei \\ National Engineering \\ Research Center for \\ Broadband Networks \& \\ Applications \\ 150 Honggu Road, Shanghai, \\ China \\ ywxu@bnc.org.cn
}

\author{
LU Xiaoyuan \\ National Engineering \\ Research Center for \\ Broadband Networks \& \\ Applications \\ 150 Honggu Road, Shanghai, \\ China \\ xylu@bnc.org.cn
}

\author{
ZHANG Yu \\ National Engineering \\ Research Center for \\ Broadband Networks \& \\ Applications \\ 150 Honggu Road, Shanghai, \\ China \\ yzhang@bnc.org.cn
}

\begin{abstract}
The structure and the architecture of the next-generation network have become the focus of recent research around the world. For looking back to see the future, this paper described the development of the China's National Testbed, which was the main mission of our striving in the last decade. We mainly presented the construction and evolution of the functional blocks of the testbed, its integration and solution$\mathrm{s}$, as well as the results of large-scale validation and industrialization promotion. As the conclusion, the future research and development directions of the national testbed were described.
\end{abstract}

\section{Categories and Subject Descriptors}

C.2.1 [Network Architecture and Design]: Network communications; C.2.3 [Network Operations]: Network management

\section{General Terms}

Design, Management

\section{Keywords}

Next Generation Network and National Service Testbed, Network Structure, Test and Demonstration, SDN

\section{INTRODUCTION}

With the development of broadband network technologies, the original IP packet switching and the most effective mechanism for Internet-based system have been unable to meet the requirements of the development of the network load, integration, and the bandwidth. Therefore, the structure and the architecture of the next-generation network have become the focus of recent research around the world. As a good platform for innovation and large-scale validation environment, testbed has become the focus of current development of the network technologies. Many countries have established their own network testbeds, such as GENI and FIND in the U.S.A, FIRE in Europe, G-LAB in Germany, JGNI II in Japan, NICTA in Australia, and etc, [4, 1, 9, 2, $14,3]$.

The development of the next generation network and national service testbed of China ( $S N G$ Testbed) started from the High Performance Broadband Information Network (3Tnet) Project in "Tenth Five-Year-Plan" and "863" key projects in the "Eleventh Five-Year-plan", which was funded by China Ministry of Science and Technology and run by the National Engineering Research Center for Broadband Networks \& Applications (where the three authors come from). Based on the SNG Testbed, various test systems have been built in China in last ten yeas, e.g., the Broadcasting Test Districts, the Telecommunications Experimental Networks, and so on. It provides an innovative network environment for the research of new systems, the testing of new technologies, the validation of new equipment, and the demonstration of new services.

\section{DEVELOPMENT PROCESS}

\subsection{The High Performance Broadband Infor- mation Network (3Tnet), 2000-2004}

The key project of "high performance broadband information network (3Tnet)" was initiated by China Ministry of Science and Technology in the "Tenth Five-Year plan" period $[7,11]$. It was a joint project of more than 50 well-known enterprises, universities and research institutes (as shown in Figure 1), including the PLA Information Engineering University, National Engineering Research Center for Broadband Networks \& Applications, Peking University, Tsinghua University, Fudan University, Shanghai Jiaotong University, Huawei, and Fiberhome Telecommunication Technologies group. With the efforts of more than 2,000 scientists, the project was successfully completed in four years time and made of a number of technical innovations.

3Tnet testbed uses the independently-developed next-generation core network technologies, such as Terabit routing, exchange and transmission, as well as the support of application environment. It covers nearly 30,000 users in the Yangtze River Delta region and the average access rate of each user is more 
than $40 \mathrm{Mb} / \mathrm{s}$, forming a testbed to provide broadband interactive services including high-definition video streaming services.

\section{Tnet Test bed}

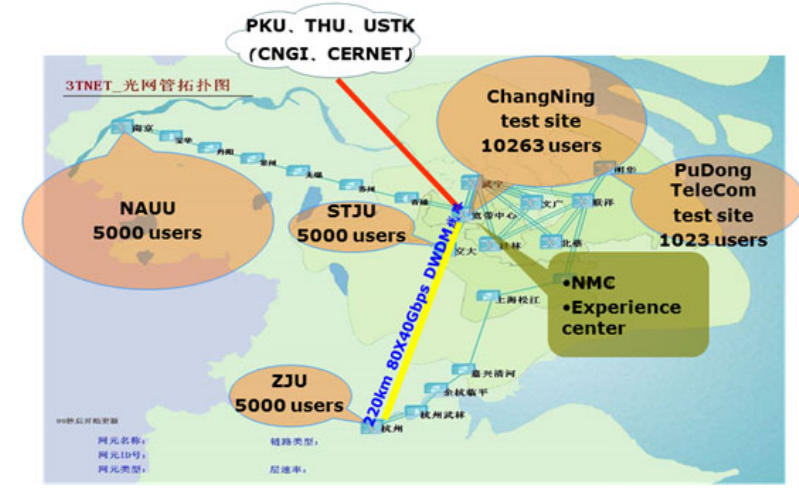

Figure 1: Topology of the High-Performance Broadband Information Network

The testbed covers 10,236 subscribers who are using FTTB + LAN in Shanghai Changning District. Apart from providing high-definition video services and broadband Internet access service, it also undertook research on the user's need$\mathrm{s}$, behavior and consumption patterns. The testbed covers 1023 FTTH users in Shanghai's Pudong district, conducting test on the integration business model of telecommunication networks and broadcasting networks. It also contains 15000 users in Shanghai Jiaotong University, Zhejiang University and Nanjing University of Aeronautics and Astronautics, carrying study on LAN performance evaluation and largescale concurrent users testing. Besides, the testbed also includes a small number of pilot users from Tsinghua University, Peking University and Chinese Academy of Sciences, doing interoperability tests with CNGI network.

Based on the Super-flat tree network architecture constituted from MB-ASON and ACR, 3Tnet testbed can support broadcast, multicast and interactive services. From the aspect of transmission technology, the project developed highspeed optical transmitters, optical receivers, loop controllers and other key modules to achieve $40 \mathrm{~Gb} / \mathrm{s}$ DWDM systems and establishing the first domestic $3.2 \mathrm{~Tb} / \mathrm{s}$ DWDM optical transmission system from Shanghai to Hangzhou. From the aspect of optical switching technology, the project achieved Tbps ASON and burst multicast technology and the evolution from ASON to MB-ASON. We have studied quick switching connections of ASON and business-driven burst scheduling, achieving the capability of $1.28 \mathrm{~T}$ at ASON node devices. On the routing technology, it supported IPV4/V6 dual stack protocol. Every node covered more than 60,000 users and the testbed supported IPTV services by multicast replication technology. Our achievements are widely recognized at home and abroad, and we won the InfoVision Award in Europe in 2006.

\subsection{China's next generation broadcasting net- works Shanghai demonstration network (NG- B) , 2008-2011}

Based on 3Tnet core technology, the next generation broadcasting network Shanghai testbed began to be built from 2008 [15]. The network consists of three parts: broadcasting test area, telecommunication test area and private network test area, as depicted in Figure 2.

The broadcasting test area covers 100,000 users, including 50000 OCN users, 20,000 cable TV users from Pudong district and 30,000 cable TV users from Jiading district. The subscribers of the private test network are from six hospitals, 10 community health service centers and a community service station in Changning district. The private test network provides online medical information service for 10,000 users.

In order to meet the requirement of two-way transformation of digital TV from the operators, we took PON + EOC networking technology to reform the original HFC network in broadcasting test area. This transformation not only kept the original HFC network but also was compatible with the existing CMTS. 1:16 optical divide ratio was set in PON network. Each EOC covered 40 users and each user can exclusively enjoy $30 \mathrm{M}$ bandwidth rather than the original $2 \mathrm{M}$ shared bandwidth of CMTS. After the transformation was completed, the network launched a variety of interactive value-added services, including HD channels, VOD, broadband Internet access, and etc.

In the private network test area, we achieved the resource sharing of medical information among various medical institutions, providing two-way interactive capabilities of residents' health documents, and supporting high-speed transmission of a large amount of medical image and video information.

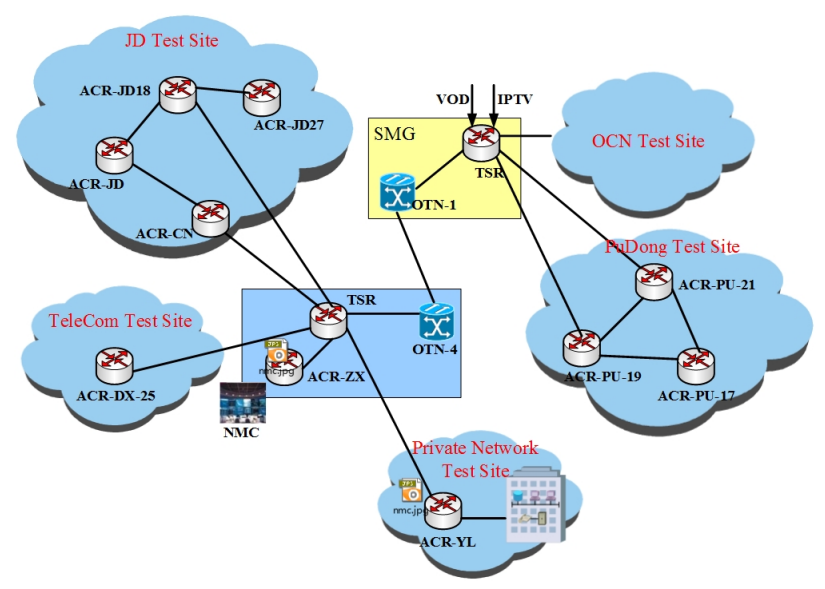

Figure 2: Topology of the NGB Testbed

The construction and testing of the NGB Shanghai testbed solved the disturbing problem when conducting fiber or Ethernet to the home. We proposed a broadband access solution by coaxial cable to the home using cable-bridge and produced EOC core equipment with 300M headend rate and achieved mass production. We successfully launched a highdefinition digital television and interactive services, successfully explored a technologically advanced and economically viable road for the construction and development of the nex- 
$\mathrm{t}$ generation broadcasting network. The testbed promoted the industrialization of the China's next generation broadcasting network.

\subsection{The next generation network and national service testbed, 2009-2013}

The Next Generation Network and National Service Testbed, as shown in Figure 3, is based on 3TNet, broadcasting test area and telecommunication test network which were built in the "Tenth five year plan" period [8]. It covers 15 cities, including Shanghai, Zhejiang province, Jiangsu province, Anhui province, Guangdong province and Hainan province. The total length of the long-haul backbone links is more than 4000 kilometers. International gates are provided.

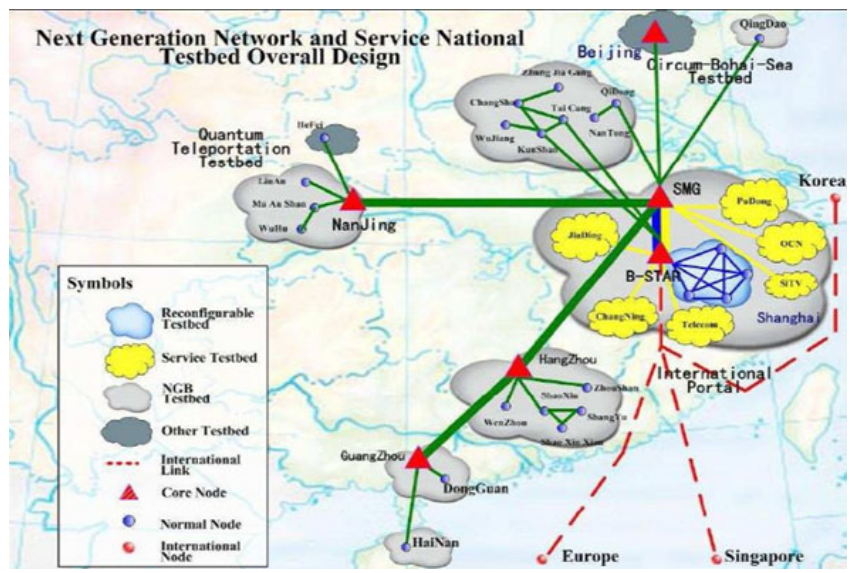

Figure 3: The Overall Structure of the Next Generation Network and National Service Testbed

One of the main tests carried out in the national testbed is the reconfigurable testbed trial (Figure 4). In the reconfigurable testbed, the optical devices are full-mesh connected and telecommunication service networks and broadcasting networks can be dynamically built based on the configuration policy and routing mode in the IP layer. Also, the transmission route of the telecommunications traffic, broadcasting traffic, congestion stream can be configured based on the policies. Telecommunication services and broadcasting networks can not only be setup separately but also be built at the same time. When they co-exist, the networks are overlapped and mutually independent.

This testbed provided an initial test and test environment for the Chinese communications industry, 863 projects and key projects. Large capacity optical networks and lots of key equipment such as flexible reconfigurable routers have been tested, validated and demonstrated in this testbed.

\section{FUTURE DEVELOPMENT}

Besides the SNG testbed, several other networks or testbed$\mathrm{s}$ are developed in China in recent years. For example, the Service-Oriented Future Internet Architecture (SOFIA) [12] takes the internet as a collection of transport channels and as a service pool and users are not required to be aware of the physical locations. The China Environment for

\section{Reconfigurable Testbed}
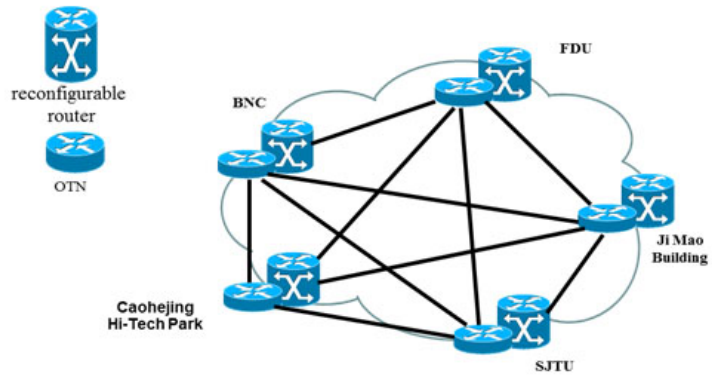

Figure 4: Topology of the Reconfigurable Testbed

Network Innovations (CENI) $[10]$ and $P \operatorname{rogrammabl} E$ virtuAl Router $\mathrm{p} L$ atform $(P E A R L)$ [13] are planning to support many types of network experiments in parallel. The $F$ uture Internet iNnovation Environment (FINE) [5] aims to achieve an open experimental environment for new protocols (including the OpenFlow protocol), architectures and researches, etc. The primary objective of the Internet Innovation Union $(I I U)[6]$ is to create a large-scale "future internet \& cutting-edge" technology-oriented experimental environment. It is expected that more innovative applications and research findings will be incubated on the "federal testbeds" platform, which was formerly known as Dragonlab.

The main differences among the SNG testbed and the above mentioned networks or testbeds (SOFIA, CENI, PEARL, FINE, IIU) are that the SNG testbed is built based on the NGB network, and its main purpose is to supply the network environment for broadcasting network and television services. In the future, the national testbed will be developed in two directions, namely SDN technology based data centers with virtual networks and 5G-based wired and wireless integrated test field.

Firstly, in order to meet the demand for the data exchange in large-scale data centers, we will study on the new generation ultra high-speed switching networks for achieving break through in key technologies, such as massive layer2 software-defined networks, multi-tenant virtualized networks, data storage and integration networks, data migration oriented layer- 2 traffic management, traffic visualized management and display. Besides, we will develop ultra high-speed TOR switches and short-range non-contact highspeed transmission core equipment. Large scale test of the new high-performance data centers will also be carried out. All these efforts will promote the demonstration of telecommunication IDC center, and accelerate industrial upgrading of broadband networks and green energy transformation development in Shanghai.

Secondly, the construction area of the wired and wireless field is located in Shanghai Pudong Science and Technology Park. Its planning area is about 5 square kilometers. It contains five bidirectional transmission base stations and two radio base stations. The targeted tests includes multi-channel heterogeneous spectrum aggregation experiments, and different-formats multi-channel wireless system 
parallel transmission technology which includes the channel establishment, activation, deactivation and demolition control process testing and the total throughput tests of multichannel parallel transmission.

\section{CONCLUSION}

This paper discussed the development process, research results and future directions of the next generation network and national service testbed. The construction and demonstration of the testbed had great significance on the network system innovation and next-generation networks tests. It will provide a good platform for testing and validating various technologies and equipment. It will also become the basis for future network development of China. We look forward to all kinds of technical communications and collaborations, especially on SDN and 5G converged network technologies.

\section{Acknowledgments}

This work is supported in part by the research on the SD$\mathrm{N}$ system with high reliability based on open architecture (13511500400) and the Shanghai SDN industrial technology and innovation strategic alliance (13DZ0510500).

\section{REFERENCES}

[1] FIND. http://www.nets-find.net.

[2] G-LAB. http://www.nets-find.net.

[3] NICTA. http://www.nicta.com.au.

[4] Berman, M., Elliott, C., and Landwebber, L. Geni: Large-scale distributed infrastructure for networking and distributed systems research. In Communications and Electronics (ICCE), 2014 IEEE Fifth International Conference on (2014), pp. 156-161.

[5] BI, J. Fine:future internet innovation environment. China Communications 01 (2015), 146-147.

[6] FAn, T., ZhaO, F., AND GaO, F. Research on cloud resource economics management model based on internet innovation union. In Cloud Computing and Intelligent Systems (CCIS), 2012 IEEE 2nd International Conference on (2012), pp. 402-406.

[7] Lin, F., Wang, X., And Xue, X. Vod service model and performance evaluation on the china's high performance broadband information network (3tnet). In Parallel and Distributed Computing, Applications and Technologies, 2005. PDCAT 2005. Sixth International Conference on (2005), pp. 163-167.

[8] Lu, X., He, L., Peng, C., And Zhang, Y. Research and demonstration of next generation network and service national testbed of china. In Testbeds and Research Infrastructures for the Development of Networks \& Communities and Workshops, 2010. TridentCom 2010. 6th International Conference on (2010), pp. 590-593.

[9] Max, L. The european fire future internet research and experimentation initiative. In Testbeds and Research Infrastructures for the Development of Networks \& Communities and Workshops, 2009. TridentCom 2009. 5th International Conference on (2009), pp. 2-3.

[10] R. Zeng. Overview of CENI Prototype. http://www.euchina-fire.eu/wp-
content/uploads/2015/01/2.CENI-EU2zengrongfei.pdf.

[11] Sun, W., Hu, W., Y.Jin, And Guo, W. Toward an optical transport network for switched digital tv distributions: the challenges and ongoing 3tnet field trial in shanghai. In Optical Internet and Next Generation Network, 2006. COIN-NGNCON 2006. The Joint International Conference on (2006), pp. 389-392.

[12] Wu, Q., Li, Z., Zhou, J., Jiang, H., Hu, Z., Liu, Y., AND XIE, G. SOFIA: toward service-oriented information centric networking. IEEE Network 28, 3 (2014), 12-18.

[13] Xie, G., He, P., Guan, H., Li, Z., Xie, Y., Luo, L., Zhang, J., Wang, Y., and Salamatian, K. PEARL: a programmable virtual router platform. IEEE Communications Magazine 49, 7 (2011), 71-77.

[14] Yamauchi, I., Miyake, T., Mikamo, Y., Shimada, J., Kobayashi, K., And Esaki, H. Jgn ii (japan gigabit network ii): A research and development system for advanced broadband networks. In Applications and the Internet Workshops, 2005. Saint Workshops 2005. The 2005 Symposium on (2005), pp. 34-37.

[15] YANG, X. An alternative to internet - the next generation broadcasting networks standard. In Information Science and Engineering (ICISE), 2010 2nd International Conference on (2010), pp. 1823-1826. 